\title{
ChemComm
}

\section{Upon the photostability of 8-nitro-cGMP and its caging as a 7-dimethylaminocoumarinyl ester $\dagger$}

Cite this: Chem. Commun., 2014, 50, 7120

Received 16th April 2014

Accepted 9th May 2014

DOI: $10.1039 / c 4 c c 02828 g$

www.rsc.org/chemcomm

8-Nitro-cGMP was recently discovered as a second messenger of nitric oxide. We describe here the synthesis and properties of DMACM-modified 8-nitro-cGMP for photochemical uncaging. Owing to the limited photostability of 8-nitro-cGMP care must be taken, but the photorelease of the intact product was readily feasible. Unexpectedly, 8-nitro-cGMP decays under formation of 8-nitrosoguanine when irradiated with light.

Cyclic nucleotides are highly abundant messenger molecules that are found in all kingdoms of life. In humans they signal and regulate a whole set of physiologically relevant processes including ion channel conductance, blood vessel relaxation, metabolism or apoptosis for instance. Cyclic nucleotides are formed as a second messenger in response to extracellular signals like peptide hormones or nitric oxide (NO). Even though cyclic nucleotides have been studied for decades, the research field is still vivid. ${ }^{1}$ In particular, the development of new methods for sensing cyclic nucleotides inside living tissues and animals drives the progress. ${ }^{2}$ Recently, Takaaki Akaike and co-workers reported 8-nitroguanosine $3^{\prime}, 5^{\prime}$-cyclic monophosphate $\mathbf{1}$ (8-nitro-cGMP) as a novel second messenger ${ }^{3}$ that is formed inside the cell in a NO-dependent manner. ${ }^{3 b}$ Interestingly, 8-nitro-cGMP can also lead to protein $S$-guanylation by reacting with active cysteine residues. ${ }^{3 f, g}$ Later, the antagonisation of 8-nitro-cGMP by hydrogen sulphide was shown, demonstrating a complex interaction of 8-nitro-cGMP with various signalling molecules. ${ }^{3 e}$ More recently, the involvement of 8-nitro cGMP in autophagy ${ }^{3 c}$ and guard cell signalling in plants ${ }^{3 d}$ was reported.

Although the cyclic nucleotide concentration inside a cell is tightly regulated, a local burst in the concentration may occur in response to extracellular signals. To study nucleotide signalling researchers have developed tools for the flash-like activation of cyclic nucleotides by light, so-called photocaged compounds.

Interfaculty Institute of Biochemistry, University of Tübingen, Auf der Morgenstelle 15, D-72076 Tübingen, Germany. E-mail: thorsten.stafforst@uni-tuebingen.de; Web: http://www.ifib.uni-tuebingen.de/research/stafforst.html; Tel: +4970712975376

$\dagger$ Electronic Supplementary Information (ESI) available: Full experimental section. See DOI: $10.1039 / \mathrm{c} 4 \mathrm{cc} 02828 \mathrm{~g}$
Light is a very attractive trigger for biological systems since it allows for the precise control over space, time and dosage. ${ }^{4}$ It is important that the caged compound does not bind to the target of the unleashed molecule prior to activation and is biologically fully inactive. This then allows us to equilibrate biological systems with the caged compound at high concentrations and to study fast processes without the need for considering slow pharmacokinetics. A very advanced and even commercially available photocaging group for cGMP and cAMP is the 7-dimethylamino-coumarinyl moiety (DMACM) that is attached to the cyclic nucleotide via a phosphoric acid ester bond. ${ }^{5}$ The respective triester is hydrolysed upon irradiation between 330 and $440 \mathrm{~nm}$ under fast release of the desired cyclic nucleotides (within 1 nano second) and coumarinyl alcohol (9). Due to the good absorption coefficient $\left(\lambda_{\max }=395 \mathrm{~nm}\right.$, $\left.\varepsilon=16 \mathrm{mM}^{-1} \mathrm{~cm}^{-1}\right)$ and high quantum yield $(\varphi=0.3)$ the uncaging is of excellent efficiency. Under physiological salt and $\mathrm{pH}$ conditions the caged compound is sufficiently soluble $(10 \mu \mathrm{M})$ and hydrolytically stable in the dark, making these compounds highly applicable. It has also been demonstrated that DMACMcaged cyclic nucleotides penetrate the mammalian cell membrane to get effective inside the cell. ${ }^{5}$

In order to study the role of 8-nitro-cGMP in signalling and to explore the $S$-guanylation of the proteome we decided to develop a photoactivatable 8-nitro-cGMP precursor carrying the DMACM group. However, in contrast to cGMP or 8-bromo-cGMP, 8-nitro-cGMP was known from the literature to absorb in the visible spectrum $\left(\lambda_{\max }=400 \mathrm{~nm}, \varepsilon=9.1 \mathrm{mM}^{-1} \mathrm{~cm}^{-1}\right){ }^{3 a}$ Since the $\mathrm{N}, \mathrm{C}$-glycosidic bond in 8-nitro-cGMP was described to be unusually long and prone to hydrolysis ${ }^{3 f}$ we expected 8-nitro-cGMP to possibly depurinate upon irradiation at $330-440 \mathrm{~nm}$, the light energy that is required for the release of the coumarinyl group. However, nothing was known about the photostability of 8-nitro-cGMP to our knowledge. To test this, we synthesised 8-nitro-cGMP from commercially available 8-bromo-cGMP by nucleophilic substitution, close to a described procedure from the literature. ${ }^{3 g}$ However, by using microwave-assisted heating we were able to accelerate the reaction from 5 days to $30 \mathrm{~h}$ and to improve the yield from $20 \%$ to $27 \%$. To test the photostability, 8-nitro-cGMP ( $27 \mu \mathrm{M}$ in degassed phosphate 
A

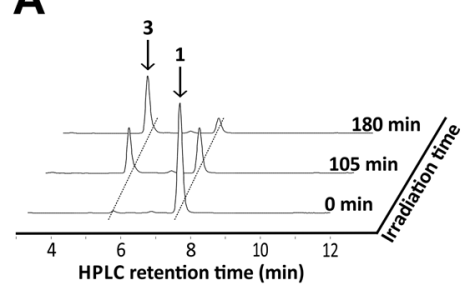

C

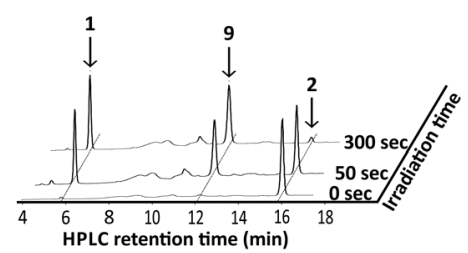

B

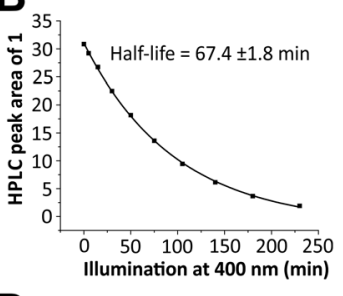

D

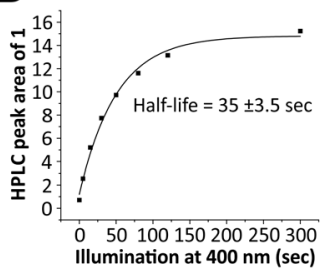

Fig. 1 Irradiation experiment with 8-nitro-cGMP (1) at $400 \mathrm{~nm}$ (top). 8-Nitro-cGMP $(27 \mu \mathrm{M})$ in degassed buffer was irradiated at $400 \mathrm{~nm}$ for $230 \mathrm{~min}$. Aliquots at various time points were subjected to HPLC analysis to monitor the decay of the starting material. (A) Selected HPLC-traces $(260 \mathrm{~nm})$ of the irradiated reaction mixture; (B) plot of the HPLC peak area values (traces at $370 \mathrm{~nm}$ ) of 1 versus irradiation time revealed a first-order half-life of $\sim 67 \mathrm{~min}$. Uncaging of DMACM-caged 8-nitro cGMP (2) at $400 \mathrm{~nm}$ (bottom). The equatorial isomer of the caged compound $(10 \mu \mathrm{M})$ in buffer was irradiated at $400 \mathrm{~nm}$ for up to $300 \mathrm{~s}$. Aliquots at various time points were subjected to HPLC analysis to monitor the formation of the 8-nitro-cGMP. (C) Selected HPLC-traces $(260 \mathrm{~nm})$ of the irradiated reaction mixture; (D) plot of the HPLC peak area values (traces at $370 \mathrm{~nm}$ ) of liberated 1 versus irradiation time revealed a first-order half-life of $\sim 35 \mathrm{~s}$.

buffer, pH 7.0) was irradiated in a fluorescence spectrometer with precise control over irradiation wavelength $(400 \pm 16 \mathrm{~nm})$ and time. ${ }^{6}$ At various time points, small aliquots were taken out and analysed by UV-spectroscopy and HPLC. As expected, 8-nitro-cGMP was unstable upon irradiation, and a new compound was formed (Fig. 1A) with a bathochromic shift of $\approx 30 \mathrm{~nm}$ in the UV-absorbance $\left(\lambda_{\max }=435 \mathrm{~nm}\right.$, Fig. S1, ESI $\left.\dagger\right)$. The half-life was determined to be $67.4 \pm 1.8 \mathrm{~min}$ (Fig. 1B) for the decomposition of the starting material. By HPLC-assisted high-resolution electrospray ionisation mass spectrometry (HPLC-HRMS) we clearly identified the constitution of the products to be $\mathrm{C}_{5} \mathrm{H}_{4} \mathrm{~N}_{6} \mathrm{O}_{2}$ and $\mathrm{C}_{5} \mathrm{H}_{9} \mathrm{O}_{8} \mathrm{P}$ (ESI, $\dagger$ supplementary spectra) that formally fits to 8-nitrosoguanine (Scheme 1A, 3), or the corresponding oxime-isomer, and to an open form (4) of ribonolactone 7 (Scheme 1A).

This was a surprise since we had expected 8-nitro-cGMP to decay via hydrolysis under release of 8-nitro-guanine. However, based on the previous reports on photodecomposition of nitrated nucleosides, ${ }^{7}$ we propose a mechanism (Scheme 1A) which involves abstraction of the $\mathrm{C}^{\prime}-\mathrm{H}$ by the excited triplet state of the nitrogroup to generate a $\mathrm{C1}^{\prime}$-radical (5) followed by the collapse of this di-radical intermediate to a five-membered cyclic structure (6) and its decomposition to ribonolactone 7. Since the analogous 2 -deoxyribonolactone is also reported to be unstable, undergoing various reactions including $\beta$-eliminations, ${ }^{7,8}$ the apparent decomposition of 7 to other products was not a surprise and may explain the failure of its direct detection. However, a hydrolysed, open form of 7 (4) could be detected with high accuracy by mass spectrometry. Due to the presence of the cyclic phosphate in
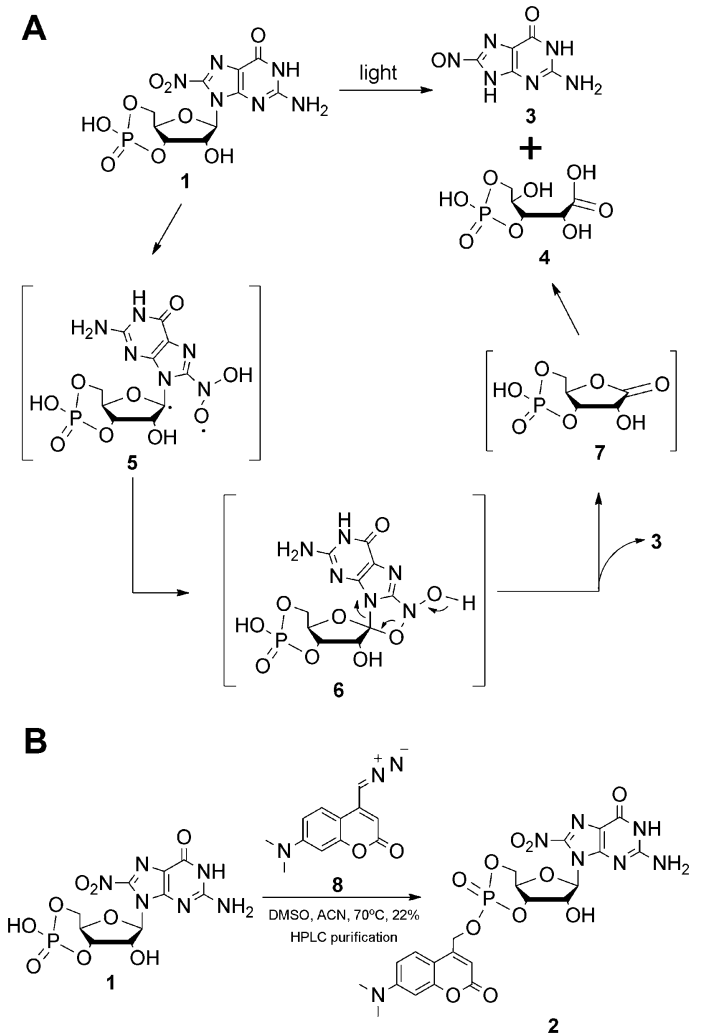

Scheme 1 (A) Proposed photodecomposition mechanism of 8-nitrocGMP; (B) synthesis of DMACM-caged 8-nitro-cGMP.

these compounds, $\beta$-elimination products of $\mathbf{4}$ or 7 cannot be discriminated by mass spectrometry and thus we are unable to clearly define the configuration of $\mathbf{4}$ or to exclude that it is a mixture of related products. Taking the stoichiometric formation of 8-nitrosoguanine (3) into account that is also supported by the appearance of two isosbestic points (410 and $310 \mathrm{~nm}$ ) in the UV spectra (Fig. S1, ESI $\dagger$ ) we exclude the possibility of the formation of $\mathbf{4}$ by direct oxidation of 5 with triplet oxygen. ${ }^{7 b}$

Even though 8-nitro-cGMP is not photostable we continued to synthesise the caged compound because the determined half-life for the photodecomposition was relatively long and it could be expected that DMACM photo-removal could possibly compete. The DMACM group was installed similarly as reported for the synthesis of DMACM-caged 8-bromo-cGMP by incubation of 8-nitro-cGMP with a diazo precursor $(8) .{ }^{5 b}$ The product was obtained in an overall yield of $22 \%$ (Scheme 1B) as a nearly 1-to-1 mixture of the axial and equatorial isomers that could be resolved during HPLC purification. First, we tested the photoactivation with the equatorial isomer. For this, the caged 8-nitro-cGMP $(\sim 10 \mu \mathrm{M}$ in phosphate buffer $\mathrm{pH} 7.0$ ) was irradiated at $400 \pm 16 \mathrm{~nm}$, very close to its absorbance maxima (Fig. S2, ESI $\dagger$ ). At various times small samples were taken out and analysed via UV-spectroscopy and HPLC. The latter clearly showed the release of the desired, intact 8-nitro-cGMP upon consumption of the caged compound (Fig. 1C). The integrity of the released 8-nitro-cGMP was also confirmed by co-injection with an authentic sample and by HPLC-HRMS analysis of the irradiation mixture (ESI, $\dagger$ supplementary spectra). First-order kinetics revealed a 
half-life of $35 \pm 3.5$ seconds for the formation of 8-nitro-cGMP (Fig. 1D). For the axial isomer we found similar first-order kinetics with a half-life of $36 \pm 2.1$ seconds (Fig. S3, ESI $\dagger$ ). Since photoactivation of the caged compound is nearly 116 -fold faster than photodecomposition of the released product, more than $95 \%$ of the caged compound can be photoactivated within 5 minutes without significant decomposition of the released cyclic nucleotide $(<5 \%)$. This is in accordance with the HPLC-data that show no detectable amounts of degraded nucleotide within the first few minutes of irradiation (Fig. 1C). Thus, photoactivation is clearly fast enough to successfully compete with product degradation. Furthermore, we determined the photochemical quantum yield $(\varphi)$ at $400 \mathrm{~nm}$ using relative methods. ${ }^{6}$ For this we compared the photoactivation-efficiency of 2 to that of a commercial sample of DMACM-caged 8-bromo-cGMP (Fig. S4, ESI $\dagger$ ). Taking an extinction coefficient of $\varepsilon=25 \mathrm{mM}^{-1} \mathrm{~cm}^{-1}$ into account, we determined $\varphi$ to be $7.5 \pm 0.4 \%$ for the axial and $7.8 \pm 0.7 \%$ for the equatorial isomer of 2. As also observed for other DMACM-caged cyclic nucleotides $\varphi$ is very similar for both isomers. ${ }^{5 b}$ In contrast, $\varphi$ was calculated to be $\sim 0.18 \%$ for the photodecomposition of 8-nitro-cGMP at $400 \mathrm{~nm}$. Since longer-wavelength light is more suitable for application in biology we also characterised the photoactivation at $430 \pm 16 \mathrm{~nm}$. Due to the reduced absorption at this wavelength slightly slower first-order kinetics were observed with $53 \pm 1.3$ seconds and $59 \pm 1.2$ seconds for the axial and equatorial isomers, respectively (Fig. S5 and S6, ESI $\dagger$ ). Compared to the uncaging kinetics of 8-nitro-cGMP, its photodegradation at $430 \mathrm{~nm}$ was stronger attenuated resulting in a half-life of $\sim 8$ hours (Fig. S7, ESI $\dagger$ ) making this wavelength even more suitable for photoactivation experiments.

The spontaneous hydrolysis of the DMACM-caged-8-nitrocGMP (2) in the dark was tested at neutral $\mathrm{pH}$ and was found to be negligible, as also reported for other DMACM-caged cyclic nucleotides (Fig. S8, ESI $\dagger$ ). ${ }^{5 b}$ With respect to their photochemical and hydrolytic properties, the two isomers of $\mathbf{2}$ are fully interchangeable - thus their resolution is dispensable for usage in biology.

For later applications it is essential to demonstrate that the caged precursor is biologically entirely inactive. To test this for DMACM-caged 8-nitro-cGMP (2), we applied an in vitro phosphorylation assay in cell extracts. This assay depends on the phosphorylation of vasodilator-stimulated phosphoprotein (VASP) by cGMP-dependent protein kinase type I (cGKI). It has been demonstrated that activation of cGKI by cGMP or 1 results in phosphorylation of VASP. ${ }^{3 g}$ For the VASP phosphorylation assay, extracts from mouse embryonic fibroblasts (MEFs) expressing $\operatorname{cGKI} \alpha^{9}$ were prepared and supplemented with either 1 or its caged precursor 2 to a final concentration of $100 \mu \mathrm{M}$. The formation of phosphorylated VASP (p-VASP) in relation to total VASP content was determined by densitometric evaluation of Western blots performed with the cell extracts. Reactions with solvent only and with $100 \mu \mathrm{M}$ cGMP served as negative and positive control, respectively. For photoactivation, samples were irradiated at $400 \pm 16 \mathrm{~nm}$ for $2 \mathrm{~min}$ (see $\mathrm{ESI} \dagger$ for details).

Both free cGMP and free 8-nitro-cGMP stimulated phosphorylation of VASP to a level of $40-45 \%$ in an irradiation-independent

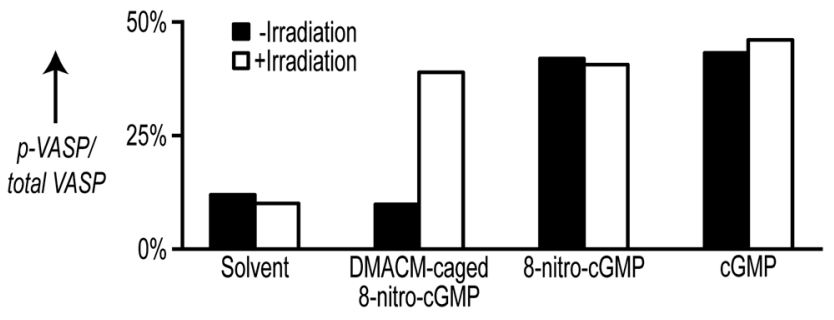

Fig. 2 Stimulation of VASP phosphorylation in cell extracts from cGKl $\alpha$ expressing MEFs supplemented with $100 \mu \mathrm{M}$ DMACM-caged 8-nitro-cGMP (2), 8-nitro-cGMP (1), or CGMP. Reactions were irradiated for $2 \mathrm{~min}$ at $400 \pm$ $16 \mathrm{~nm}$ and then incubated for $20 \mathrm{~min}$ in the dark. The amount of $\mathrm{p}$-VASP and total VASP was estimated by densitometric analysis of Western blots probed with VASP antibodies.

manner (Fig. 2). In contrast, addition of the caged precursor 2 without irradiation caused no VASP phosphorylation. However, irradiating the reaction supplemented with 2 yielded p-VASP levels comparable to that obtained with free cGMP or 1 (Fig. 2). To test if VASP phosphorylation was indeed caused by cGKI $\alpha$, we performed additional VASP phosphorylation assays with cell extracts derived from cGKI-deficient $\mathrm{MEFs}^{9}$ (Fig. S9, $\mathrm{ESI}^{\dagger}$ ). In the absence of cGKI, none of the tested compounds increased VASP phosphorylation above control levels in the presence of solvent nor did irradiation show considerable influence on p-VASP levels. However, addition of purified recombinant cGKI $\alpha$ enzyme restored VASP phosphorylation upon addition of the tested compounds (Fig. S9, ESI $\dagger$ ). Together, these data show that, while free 8-nitro-cGMP is able to activate cGKI $\alpha$ to a similar extent as cGMP, this action of 8-nitro-cGMP gets completely blocked by incorporation of the DMACM group and can be efficiently re-activated by light.

In conclusion, we have demonstrated that the DMACM group is well suited for caging 8-nitro-cGMP even though 8-nitro-cGMP itself is not photostable at the required activation wavelength. With respect to photoactivation efficiency, solubility and hydrolytic stability, the caging group behaves similar and thus equally beneficial compared to other reported DMACM-caged cyclic nucleotides. Moreover, we found 8-nitro-cGMP to photodegrade via an unexpected route. Notably, the introduction of the DMACM caging group completely inactivated the biological activity of 1 in a phosphorylation assay suggesting that our caged 8-nitro-cGMP is a valuable tool for signalling studies.

The generous support from DFG (STA 1053/3-1, FE 438/2-4), Fonds der Chemischen Industrie and the University of Tübingen is gratefully acknowledged. The authors thank P. Ringer and B. Birk for the experimental support.

\section{Notes and references}

1 (a) J. A. Beavo and L. L. Brunton, Nat. Rev. Mol. Cell Biol., 2002, 3, 710; (b) B. Kemp-Harper and R. Feil, Sci. Signaling, 2008, 1, pe12.

2 M. Thunemann, L. Wen, M. Hillenbrand, A. Vachaviolos, S. Feil, T. Ott, X. Han, D. Fukumura, R. K. Jain, M. Russwurm, C. de Wit and R. Feil, Circ. Res., 2013, 113, 365.

3 (a) T. Akaike, S. Okamoto, T. Sawa, J. Yoshitake, F. Tamura, K. Ichimori, K. Miyazaki, K. Sasamoto and H. Maeda, Proc. Natl. Acad. Sci. U. S. A., 2003, 100, 685; (b) S. Fujii, T. Sawa, H. Ihara, K. I. Tong, T. Ida, T. Okamoto, A. K. Ahtesham, Y. Ishima, H. Motohashi, M. Yamamoto and T. Akaike, J. Biol. Chem., 2010, 
285, 23970; (c) C. Ito, Y. Saito, T. Nozawa, S. Fujii, T. Sawa, H. Inoue, T. Matsunaga, S. Khan, S. Akashi, R. Hashimoto, C. Aikawa, E. Takahashi, H. Sagara, M. Komatsu, K. Tanaka, T. Akaike, I. Nakagawa and H. Arimoto, Mol. Cell, 2013, 52, 794; $(d)$ T. Joudoi, Y. Shichiri, N. Kamizono, T. Akaike, T. Sawa, J. Yoshitake, N. Yamada and S. Iwai, Plant Cell, 2013, 25, 558; (e) M. Nishida, T. Sawa, N. Kitajima, K. Ono, H. Inoue, H. Ihara, H. Motohashi, M. Yamamoto, M. Suematsu, H. Kurose, A. van der Vliet, B. A. Freeman, T. Shibata, K. Uchida, Y. Kumagai and T. Akaike, Nat. Chem. Biol., 2012, 8, 714; $(f)$ Y. Saito, H. Taguchi, S. Fujii, T. Sawa, E. Kida, C. Kabuto, T. Akaike and H. Arimoto, Chem. Commun., 2008, 5984; $(g)$ T. Sawa, M. H. Zaki, T. Okamoto, T. Akuta, Y. Tokutomi, S. Kim-Mitsuyama, H. Ihara, A. Kobayashi, M. Yamamoto, S. Fujii, H. Arimoto and T. Akaike, Nat. Chem. Biol., 2007, 3, 727.
4 (a) C. Brieke, F. Rohrbach, A. Gottschalk, G. Mayer and A. Heckel, Angew. Chem., Int. Ed., 2012, 51, 8446; (b) G. Mayer and A. Heckel, Angew. Chem., Int. Ed., 2006, 45, 4900.

5 (a) V. Hagen, J. Bendig, S. Frings, T. Eckardt, S. Helm, D. Reuter and U. B. Kaupp, Angew. Chem., Int. Ed., 2001, 40, 1046; (b) V. Hagen, S. Frings, B. Wiesner, S. Helm, U. B. Kaupp and J. Bendig, ChemBioChem, 2003, 4, 434.

6 T. Stafforst and J. M. Stadler, Angew. Chem., Int. Ed., 2013, 52, 12448. 7 (a) C. Crey-Desbiolles, J. Lhomme, P. Dumy and M. Kotera, J. Am. Chem. Soc., 2004, 126, 9532; (b) M. Kotera, Y. Roupioz, E. Defrancq, A. G. Bourdat, J. Garcia, C. Coulombeau and J. Lhomme, Chem. - Eur. J., 2000, 6, 4163.

8 H. J. Lenox, C. P. McCoy and T. L. Sheppard, Org. Lett., 2001, 3, 2415.

9 P. M. Muller, R. Gnugge, S. Dhayade, M. Thunemann, P. KrippeitDrews, G. Drews and R. Feil, Free Radical Biol. Med., 2012, 53, 1574. 